\title{
UNICITY THEOREMS FOR ENTIRE FUNCTIONS CONCERNING FOUR SMALL FUNCTIONS
}

\author{
HONG-XUN YI
}

\begin{abstract}
This paper studies the problem of uniqueness of entire functions concerning four small functions and shows that if two entire functions $f$ and $g$ satisfy $\bar{E}\left(a_{j}, k, f\right)=$ $\bar{E}\left(a_{j}, k, g\right)$ for $j=1,2,3,4$, where $a_{j}$ are four distinct small functions with respect to $f$ and $g$, and $k$ is a positive integer or $\infty$ with $k \geq 8$, then $f \equiv g$.
\end{abstract}

\section{Introduction and main result}

In this paper, by meromorphic function we shall always mean a meromorphic function in the complex plane $\boldsymbol{C}$. We adopt the standard notations in the Nevanlinna theory of meromorphic functions as explained in [1]. For any nonconstant meromorphic function $f(z)$, we denote by $S(r, f)$ any quantity satisfying $S(r, f)=o(T(r, f))$ as $r \rightarrow \infty$ except possibly for a set of $r$ of finite linear measure. A meromorphic function $a(z)$ is called a small function with respect to $f(z)$ if $T(r, a)=S(r, f)$. Let $S(f)$ be the set of meromorphic functions in the complex plane $C$ which are small functions with respect to $f$. Note that $C \in S(f)$ and $S(f)$ is a field (see [2]).

Let $f(z)$ be a nonconstant entire function, $a(z) \in S(f)$, and let $k$ be a positive integer or $\infty$. We denote by $\bar{E}(a, k, f)$ the set of distinct zeros of $f(z)-a(z)$ with multiplicities $\leq k$ (see [3]). In particular, we denote by $\bar{E}(a, \infty, f)$ the set of distinct zeros of $f(z)-a(z)$.

Let $f(z)$ and $g(z)$ be nonconstant entire functions and let $a(z) \in S(f) \cap$ $S(g)$. We denote by $\bar{N}_{0}(r, a, f, g)$ the counting function of common zeros of $f(z)-a(z)=0$ and $g(z)-a(z)=0$ (ignoring multiplicities), each point counted only once. Let

$$
\bar{N}_{12}(r, a, f, g):=\bar{N}(r, a, f)+\bar{N}(r, a, g)-2 \bar{N}_{0}(r, a, f, g),
$$

then $\bar{N}_{12}(r, a, f, g)$ denotes the counting function of different solutions to $f(z)-$ $a(z)=0$ and $g(z)-a(z)=0$. Set

2000 Mathematics Subject Classification: 30D35, 30D20.

Key words and phrases: Entire function, small function, unicity theorem.

Project supported by the NSFC (No. 19871050) and the RFDP (No. 98042209).

Received September 21, 2000; revised April 16, 2001. 


$$
\lambda(a, f, g):=1-\limsup _{r \rightarrow \infty} \frac{\bar{N}_{12}(r, a, f, g)}{T(r, f)+T(r, g)} .
$$

Obviously, $0 \leq \lambda(a, f, g) \leq 1$ (see [4]).

If

$$
\bar{N}(r, a, f)-\bar{N}_{0}(r, a, f, g)=0 \quad \text { and } \quad \bar{N}(r, a, g)-\bar{N}_{0}(r, a, f, g)=0,
$$

we say $f(z)$ and $g(z)$ share $a(z)$ IM. If

$$
\bar{N}(r, a, f)-\bar{N}_{0}(r, a, f, g)=S(r, f) \quad \text { and } \quad \bar{N}(r, a, g)-\bar{N}_{0}(r, a, f, g)=S(r, g),
$$

we say $f(z)$ and $g(z)$ share $a(z)$ "IM" (see [5]). It is obvious that if $f(z)$ and $g(z)$ share $a(z) \mathrm{IM}$, then $\bar{E}(a, \infty, f)=\bar{E}(a, \infty, g)$ and $\bar{N}_{12}(r, a, f, g)=0$; if $f(z)$ and $g(z)$ share $a(z)$ "IM", then $\bar{N}_{12}(r, a, f, g)=S(r, f)+S(r, g)$.

Recently, B. Li [6] and independently Y. Li [7] proved the following theorem:

THEOREM A. If $f$ and $g$ are nonconstant entire functions sharing $a_{j}$ IM for $j=1,2,3,4$, where $a_{1}, a_{2}, a_{3}, a_{4}$ are four distinct elements in $S(f) \cap S(g)$, then $f \equiv g$.

In this paper, we deal with the problem of uniqueness of entire functions concerning four small functions. We shall deal with the problem of uniqueness of meromorphic functions concerning five small functions in another paper. In this paper, we prove the following results which are improvements of Theorem A:

THEOREM 1.1. Let $f$ and $g$ be nonconstant entire functions and let $a_{j}$ $(j=1,2,3,4)$ be four distinct elements in $S(f) \cap S(g)$. If

$$
\bar{E}\left(a_{j}, k, f\right)=\bar{E}\left(a_{j}, k, g\right) \quad(j=1,2,3,4),
$$

where $k$ is a positive integer or $\infty$ with $k \geq 8$, then $f \equiv g$.

Remark. In Theorem 1.1, we do not suppose that $f$ and $g$ always share $a_{j}(j=1,2,3,4)$ IM. In Theorem A, we suppose that $f$ and $g$ share $a_{j}(j=1$, $2,3,4) \mathrm{IM}$, and hence

$$
\bar{E}\left(a_{j}, k, f\right)=\bar{E}\left(a_{j}, k, g\right) \quad(j=1,2,3,4),
$$

where $k=\infty$. In Theorem $1.1, k \geq 8$, from this we know that Theorem 1.1 is an improvement of Theorem A.

THEOREM 1.2. Let $f$ and $g$ be nonconstant entire functions and let $a_{j}(j=1$, $2,3,4)$ be four distinct elements in $S(f) \cap S(g)$. If $f$ and $g$ share $a_{j}(j=1,2,3)$ "IM", and

$$
\bar{E}\left(a_{4}, k, f\right)=\bar{E}\left(a_{4}, k, g\right),
$$

where $k$ is a positive integer or $\infty$ with $k \geq 3$, then $f \equiv g$. 
THEOREM 1.3. Let $f$ and $g$ be nonconstant entire functions and let $a_{j}(j=1$, $2,3,4)$ be four distinct elements in $S(f) \cap S(g)$. If $f$ and $g$ share $a_{j}(j=1,2,3)$ "IM", and

$$
\lambda\left(a_{4}, f, g\right)>\frac{2}{3},
$$

then $f \equiv g$.

Remark. In Theorem 1.3, we do not suppose that $f$ and $g$ always share $a_{4}$ IM. In Theorem A, we suppose that $f$ and $g$ share $a_{4} \mathrm{IM}$, and hence $\lambda\left(a_{4}, f, g\right)=$ 1 , from this we know that Theorem 1.3 is an improvement of Theorem A.

\section{Some lemmas}

LEMma 2.1. Let $h$ be a nonconstant entire function, and let $a \in S(h)$ and $a \not \equiv 0 . \quad$ Then

$$
a^{\prime} h-a h^{\prime} \not \equiv 0
$$

and

$$
\begin{aligned}
& m\left(r, \frac{a^{\prime} h-a h^{\prime}}{h-a}\right)=S(r, h), \\
& m\left(r, \frac{a^{\prime} h-a h^{\prime}}{h(h-a)}\right)=S(r, h) .
\end{aligned}
$$

Proof. Suppose that

$$
a^{\prime} h-a h^{\prime} \equiv 0 .
$$

By integration we have

$$
a \equiv c h,
$$

where $c$ is a nonzero constant. From this we get

$$
T(r, a)=T(r, h)+O(1),
$$

which contradicts $a \in S(h)$. Thus

$$
a^{\prime} h-a h^{\prime} \not \equiv 0 .
$$

From the following equalities

$$
\begin{aligned}
& \frac{a^{\prime} h-a h^{\prime}}{h-a}=a^{\prime}-\frac{a\left(h^{\prime}-a^{\prime}\right)}{h-a}, \\
& \frac{a^{\prime} h-a h^{\prime}}{h(h-a)}=\frac{h^{\prime}}{h}-\frac{h^{\prime}-a^{\prime}}{h-a},
\end{aligned}
$$

we obtain (2.1) and (2.2). 
LEMMA 2.2. Let $f$ and $g$ be nonconstant entire functions and let $a_{1}, a_{2}, a_{3}, a_{4}$ be four distinct elements in $S(f) \cap S(g)$. If $f \not \equiv g$, then

$$
\begin{aligned}
\bar{N}_{0}\left(r, a_{4}, f, g\right) \leq & \sum_{j=1}^{3} \bar{N}_{12}\left(r, a_{j}, f, g\right)+S(r, f)+S(r, g), \\
\bar{N}\left(r, a_{4}, f\right)+\bar{N}\left(r, a_{4}, g\right) \leq & 2 \sum_{j=1}^{4} \bar{N}_{12}\left(r, a_{j}, f, g\right)-\bar{N}_{12}\left(r, a_{4}, f, g\right) \\
& +S(r, f)+S(r, g) .
\end{aligned}
$$

The proof of Lemma 2.2 is given in $\$ 3$.

LEMma 2.3 (see [1]). Let $f(z)$ be a nonconstant meromorphic function and let $a_{1}, a_{2}, a_{3}$ be three distinct elements in $S(f) \cup\{\infty\}$. Then

$$
T(r, f) \leq \sum_{j=1}^{3} \bar{N}\left(r, a_{j}, f\right)+S(r, f) .
$$

Let $f(z)$ be a nonconstant entire function, $a(z) \in S(f)$, and $k$ be a positive integer. We denote by $\bar{N}_{k)}(r, a, f)$ the counting function of distinct zeros of $f(z)-a(z)$ with multiplicities $\leq k$, by $\bar{N}_{(k+1}(r, a, f)$ the counting function of distinct zeros of $f(z)-a(z)$ with multiplicities $\geq k+1$, each point in these counting functions is counted only once (see [3]).

LEMMA 2.4. Let $f(z)$ be a nonconstant entire function and let $a_{1}, a_{2}$ be two distinct elements in $S(f)$, and $k$ be a positive integer. Then

$$
\begin{gathered}
T(r, f) \leq \sum_{j=1}^{2} \bar{N}\left(r, a_{j}, f\right)+S(r, f), \\
\sum_{j=1}^{2} \bar{N}_{(k+1}\left(r, a_{j}, f\right) \leq \frac{1}{k} T(r, f)+S(r, f) .
\end{gathered}
$$

Proof. Noting that $f(z)$ is entire function, from Lemma 2.3, we can obtain (2.7). Noting that for $j=1,2$

$$
k \bar{N}_{(k+1}\left(r, a_{j}, f\right)+\bar{N}\left(r, a_{j}, f\right) \leq N\left(r, a_{j}, f\right) \leq T(r, f)+S(r, f),
$$

we have from $(2.7)$

$$
k \sum_{j=1}^{2} \bar{N}_{(k+1}\left(r, a_{j}, f\right)+T(r, f) \leq 2 T(r, f)+S(r, f) .
$$

From this we get (2.8). 
LEMMA 2.5. Let $f(z)$ be a nonconstant entire function and let $a_{1}, a_{2}, a_{3}$ be three distinct elements in $S(f)$, and $k$ be a positive integer. Then

$$
\begin{gathered}
\frac{3}{2} T(r, f) \leq \sum_{j=1}^{3} \bar{N}\left(r, a_{j}, f\right)+S(r, f), \\
\sum_{j=1}^{3} \bar{N}_{(k+1}\left(r, a_{j}, f\right) \leq \frac{3}{2 k} T(r, f)+S(r, f) .
\end{gathered}
$$

Proof. By Lemma 2.4, we have

$$
\begin{aligned}
& T(r, f) \leq \bar{N}\left(r, a_{1}, f\right)+\bar{N}\left(r, a_{2}, f\right)+S(r, f), \\
& T(r, f) \leq \bar{N}\left(r, a_{2}, f\right)+\bar{N}\left(r, a_{3}, f\right)+S(r, f), \\
& T(r, f) \leq \bar{N}\left(r, a_{3}, f\right)+\bar{N}\left(r, a_{1}, f\right)+S(r, f) .
\end{aligned}
$$

From the above we get (2.9). Again by Lemma 2.4, we have

$$
\begin{aligned}
& \bar{N}_{(k+1}\left(r, a_{1}, f\right)+\bar{N}_{(k+1}\left(r, a_{2}, f\right) \leq \frac{1}{k} T(r, f)+S(r, f), \\
& \bar{N}_{(k+1}\left(r, a_{2}, f\right)+\bar{N}_{(k+1}\left(r, a_{3}, f\right) \leq \frac{1}{k} T(r, f)+S(r, f), \\
& \bar{N}_{(k+1}\left(r, a_{3}, f\right)+\bar{N}_{(k+1}\left(r, a_{1}, f\right) \leq \frac{1}{k} T(r, f)+S(r, f) .
\end{aligned}
$$

From the above we get (2.10).

LEMma 2.6. Let $f(z)$ be a nonconstant entire function and let $a_{1}, a_{2}, a_{3}, a_{4}$ be four distinct elements in $S(f)$, and $k$ be a positive integer. Then

$$
\begin{gathered}
2 T(r, f) \leq \sum_{j=1}^{4} \bar{N}\left(r, a_{j}, f\right)+S(r, f), \\
\sum_{j=1}^{4} \bar{N}_{(k+1}\left(r, a_{j}, f\right) \leq \frac{2}{k} T(r, f)+S(r, f) .
\end{gathered}
$$

Proof. By Lemma 2.4, we have

$$
\begin{aligned}
& T(r, f) \leq \bar{N}\left(r, a_{1}, f\right)+\bar{N}\left(r, a_{2}, f\right)+S(r, f), \\
& T(r, f) \leq \bar{N}\left(r, a_{3}, f\right)+\bar{N}\left(r, a_{4}, f\right)+S(r, f) .
\end{aligned}
$$

From the above we get (2.11). Again by Lemma 2.4, we have 


$$
\begin{aligned}
& \bar{N}_{(k+1}\left(r, a_{1}, f\right)+\bar{N}_{(k+1}\left(r, a_{2}, f\right) \leq \frac{1}{k} T(r, f)+S(r, f), \\
& \bar{N}_{(k+1}\left(r, a_{3}, f\right)+\bar{N}_{(k+1}\left(r, a_{4}, f\right) \leq \frac{1}{k} T(r, f)+S(r, f) .
\end{aligned}
$$

From the above we get (2.12).

\section{Proof of Lemma 2.2}

If $\bar{N}_{0}\left(r, a_{4}, f, g\right)=S(r, f)+S(r, g),(2.5)$ holds obviously. In the following we suppose

$$
\bar{N}_{0}\left(r, a_{4}, f, g\right) \neq S(r, f)+S(r, g) .
$$

Set

$$
L(w):=\frac{w-a_{1}}{a_{2}-a_{1}} .
$$

Let $F(z):=L(f(z)), G(z):=L(g(z)), b_{j}:=L\left(a_{j}\right) \quad(j=1,2,3,4)$. From (3.2) $b_{1}$, $b_{2}, b_{3}, b_{4}$ are distinct and $b_{1}=0, b_{2}=1$,

$$
T(r, F)=T(r, f)+S(r, f), \quad T(r, G)=T(r, g)+S(r, g),
$$

and $b_{3}, b_{4} \in S(F) \cap S(G)$. Noting $f \not \equiv g$, we have

$$
F \not \equiv G \text {. }
$$

From (3.1) and (3.3), we get

$$
\bar{N}_{0}\left(r, b_{4}, F, G\right) \neq S(r, F)+S(r, G) .
$$

Set

$$
H:=\frac{F^{\prime}\left(a^{\prime} G-a G^{\prime}\right)(F-G)}{F(F-1) G(G-a)}-\frac{G^{\prime}\left(a^{\prime} F-a F^{\prime}\right)(F-G)}{G(G-1) F(F-a)},
$$

where $a=b_{3}$. Then we have from (3.6)

$$
H=\frac{(F-G) Q}{F(F-1)(F-a) G(G-1)(G-a)},
$$

where

$$
\begin{aligned}
Q= & F^{\prime}\left(a^{\prime} G-a G^{\prime}\right)(G-1)(F-a)-G^{\prime}\left(a^{\prime} F-a F^{\prime}\right)(F-1)(G-a) \\
= & a^{\prime} F F^{\prime} G^{2}-a^{\prime} F F^{\prime} G-a(a-1) F F^{\prime} G^{\prime}-a a^{\prime} F^{\prime} G^{2}+a a^{\prime} F^{\prime} G \\
& -a^{\prime} F^{2} G G^{\prime}+a^{\prime} F G G^{\prime}+a(a-1) F^{\prime} G G^{\prime}+a a^{\prime} F^{2} G^{\prime}-a a^{\prime} F G^{\prime} .
\end{aligned}
$$

Suppose that $H \equiv 0$. From (3.4) and (3.6) we obtain

$$
\frac{F^{\prime}\left(a^{\prime} G-a G^{\prime}\right)}{(F-1)(G-a)} \equiv \frac{G^{\prime}\left(a^{\prime} F-a F^{\prime}\right)}{(G-1)(F-a)} .
$$


If $a$ is a constant, from (3.4) and (3.9) we get $a=1$, which is a contradiction. Thus, $a$ is not a constant. By Lemma 2.1,

$$
a^{\prime} F-a F^{\prime} \not \equiv 0 .
$$

From (3.9) we have

$$
\frac{F^{\prime}\left(a^{\prime} G-a G^{\prime}\right)}{G^{\prime}\left(a^{\prime} F-a F^{\prime}\right)}-1 \equiv \frac{(F-1)(G-a)}{(G-1)(F-a)}-1 .
$$

Thus,

$$
\frac{a^{\prime}\left[\left(F^{\prime}-G^{\prime}\right) G-(F-G) G^{\prime}\right]}{G^{\prime}\left(a^{\prime} F-a F^{\prime}\right)} \equiv \frac{(1-a)(F-G)}{(G-1)(F-a)} .
$$

From this we get

$$
\frac{F^{\prime}-G^{\prime}}{F-G} \equiv \frac{(1-a) G^{\prime}\left(a^{\prime} F-a F^{\prime}\right)}{a^{\prime} G(G-1)(F-a)}+\frac{G^{\prime}}{G} .
$$

By (3.5), we know that there is a point $z_{0}$ such that $z_{0}$ is a common zero of $F-b_{4}$ and $G-b_{4}$ that is not a zero or a pole of $a, a^{\prime}, b_{4}, b_{4}-1, b_{4}-a$. It is obvious that $z_{0}$ is a pole of the left-hand side of (3.10), and not a pole of the right-hand side of (3.10), which is a contradiction. Thus,

$$
H \not \equiv 0 \text {. }
$$

From (3.7) and (3.11), we have

$$
\begin{aligned}
\bar{N}_{0}\left(r, b_{4}, F, G\right) & \leq N(r, 0, H)+S(r, F)+S(r, G) \\
& \leq m(r, H)+N(r, H)+S(r, F)+S(r, G) .
\end{aligned}
$$

From (3.6) we have

$$
\begin{aligned}
H= & \frac{F^{\prime}}{F-1} \cdot \frac{a^{\prime} G-a G^{\prime}}{G(G-a)}-\left(\frac{F^{\prime}}{F-1}-\frac{F^{\prime}}{F}\right) \cdot \frac{a^{\prime} G-a G^{\prime}}{G-a} \\
& -\left(\frac{G^{\prime}}{G-1}-\frac{G^{\prime}}{G}\right) \cdot \frac{a^{\prime} F-a F^{\prime}}{F-a}+\frac{G^{\prime}}{G-1} \cdot \frac{a^{\prime} F-a F^{\prime}}{F(F-a)} .
\end{aligned}
$$

Again by Lemma 2.1 we obtain

$$
m(r, H)=S(r, F)+S(r, G) .
$$

Next we make an estimate of $N(r, H)$.

By (3.6) we know that the poles of $H$ occur possibly only from the zeros of $F, G, F-1, G-1, F-a$ and $G-a$, and the poles of $a$. Let $S_{0}$ be the set of all zeros, 1-points and poles of $a$ and let for $j=1,2,3$

$$
A_{j}:=\left\{z \mid F(z)-b_{j}(z)=0\right\} \backslash S_{0}, \quad B_{j}:=\left\{z \mid G(z)-b_{j}(z)=0\right\} \backslash S_{0},
$$


where $b_{1}=0, b_{2}=1$ and $b_{3}=a$. Thus, the poles of $H$ occur possibly only from the set

$$
\left(\bigcup_{1 \leq p \leq 3} A_{p}\right) \cup\left(\bigcup_{1 \leq q \leq 3} B_{q}\right) \cup S_{0} .
$$

Let

$$
\begin{aligned}
& S_{1}:=\bigcup_{1 \leq p \leq 3}\left\{A_{p} \cap B_{p}\right\}, \\
& S_{2}:=\left\{\bigcup_{1 \leq p \leq 3} A_{p}\right\} \backslash\left\{\bigcup_{1 \leq q \leq 3} B_{q}\right\}, \\
& S_{3}:=\left\{\bigcup_{1 \leq q \leq 3} B_{q}\right\} \backslash\left\{\bigcup_{1 \leq p \leq 3} A_{p}\right\}, \\
& S_{4}:=\bigcup_{\substack{1 \leq p \leq 3 \\
1 \leq q \leq 3 \\
p \neq q}}\left\{A_{p} \cap B_{q}\right\} .
\end{aligned}
$$

It is clear that $S_{1}$ is the set of common zeros of $F-b_{j}$ and $G-b_{j}(j=1,2,3)$; $S_{2}$ is the set of zeros of $F-b_{j}(j=1,2,3)$ which are not zeros of $G-b_{k}$ $(k=1,2,3) ; S_{3}$ is the set of zeros of $G-b_{j}(j=1,2,3)$ which are not zeros of $F-b_{k}(k=1,2,3) ; S_{4}$ is the set of zeros of $F-b_{j}$ which are zeros of $G-b_{k}$, where $1 \leq j, k \leq 3$ and $j \neq k$. From this we have

$$
\bigcup_{1 \leq j \leq 4} S_{j}=\left(\bigcup_{1 \leq p \leq 3} A_{p}\right) \cup\left(\bigcup_{1 \leq q \leq 3} B_{q}\right) .
$$

Thus, the poles of $H$ occur possibly only from the set

$$
\left(\bigcup_{1 \leq j \leq 4} S_{j}\right) \cup S_{0} \text {. }
$$

Since $a \in S(F) \cap S(G)$, the contribution of $S_{0}$ to $N(r, H)$ is at most $S(r, F)+$ $S(r, G)$. We next proceed to estimate the contribution of $\bigcup_{1 \leq j \leq 4} S_{j}$ to $N(r, H)$. We discuss the following four cases:

CASE 1. The contribution of $S_{1}$ to $N(r, H)$.

We distinguish the following three subcases:

SubCASE 1.1. Suppose that $z_{11} \in A_{1} \cap B_{1}$, and assume that $z_{11}$ is a zero of $F$ of order $p_{1}$ and $G$ of order $q_{1}$, from (3.8), we know that $z_{11}$ is a zero of $Q$ of order at least $p_{1}+q_{1}-1$. Noting that $z_{11}$ is a zero of $F-G$, from (3.7) we know that $z_{11}$ is not a pole of $H$.

SubCASE 1.2. Suppose that $z_{12} \in A_{2} \cap B_{2}$. Noting that $z_{12}$ is a zero of $F-G$, 
a simple pole of $F^{\prime} /(F-1)$ and $G^{\prime} /(G-1)$, from (3.6) we know that $z_{12}$ is not a pole of $H$.

SubCASE 1.3. Suppose that $z_{13} \in A_{3} \cap B_{3}$, from (2.4), we know that $z_{13}$ is a simple pole of $\left(a^{\prime} F-a F^{\prime}\right) /(F(F-a))$ and $\left(a^{\prime} G-a G^{\prime}\right) /(G(G-a))$. Noting that $z_{13}$ is a zero of $F-G$, from (3.6) we know that $z_{13}$ is not a pole of $H$.

From the above, we know that the points in $S_{1}$ are not poles of $H$. Thus, the contribution of $S_{1}$ to $N(r, H)$ is 0 .

CASE 2. The contribution of $S_{2}$ to $N(r, H)$.

We distinguish the following three subcases:

SubCASE 2.1. Suppose that $z_{21} \in A_{1}$ and $z_{21} \notin \bigcup_{1 \leq q \leq 3} B_{q}$. Then $z_{21}$ is a zero of $F$, not a zero of $G, G-1$ and $G-a$. From (3.6), we know that $z_{21}$ is a pole of $H$ of order at most 1 .

SubCASE 2.2. Suppose that $z_{22} \in A_{2}$ and $z_{22} \notin \bigcup_{1 \leq q \leq 3} B_{q}$. Then $z_{22}$ is a zero of $F-1$, not a zero of $G, G-1$ and $G-a$. From (3.6), we know that $z_{22}$ is a pole of $H$ of order at most 1 .

SubCase 2.3. Suppose that $z_{23} \in A_{3}$ and $z_{23} \notin \bigcup_{1 \leq q \leq 3} B_{q}$. Then $z_{23}$ is a zero of $F-a$, not a zero of $G, G-1$ and $G-a$. From (2.4) and (3.6), we know that $z_{23}$ is a pole of $H$ of order at most 1 .

From the above, we know that the points in $S_{2}$ are poles of $H$ of order at most 1 .

CASE 3. The contribution of $S_{3}$ to $N(r, H)$.

Similar to Case 2, we can obtain that the points in $S_{3}$ are poles of $H$ of order at most 1 .

CaSe 4. The contribution of $S_{4}$ to $N(r, H)$.

Suppose that $z_{4} \in S_{4}$. Then $z_{4} \in A_{p}$ and $z_{4} \in B_{q}$, where $1 \leq p, q \leq 3$ and $p \neq q$. Without loss of generality we can assume that $z_{4} \in A_{1}$ and $z_{4} \in B_{2}$. Then, $z_{4}$ is a zero of $F$ and $G-1$. From (3.6), we know that $z_{4}$ is a pole of $H$ of order at most 2. Thus, the points in $S_{4}$ are poles of $H$ of order at most 2.

Noting that each point of $S_{2}$ and $S_{3}$ is counted once, each point of $S_{4}$ is counted twice in

$$
\sum_{j=1}^{3} \bar{N}_{12}\left(r, b_{j}, F, G\right),
$$

from the above we obtain that the contribution of $\bigcup_{1 \leq j \leq 4} S_{j}$ to $N(r, H)$ is at most

$$
\sum_{j=1}^{3} \bar{N}_{12}\left(r, b_{j}, F, G\right) .
$$


Thus,

$$
N(r, H) \leq \sum_{j=1}^{3} \bar{N}_{12}\left(r, b_{j}, F, G\right)+S(r, F)+S(r, G) .
$$

Substituting (3.13) and (3.14) into (3.12) we derive

$$
\bar{N}_{0}\left(r, b_{4}, F, G\right) \leq \sum_{j=1}^{3} \bar{N}_{12}\left(r, b_{j}, F, G\right)+S(r, F)+S(r, G) .
$$

From this we get (2.5). Noting that

$$
\bar{N}\left(r, a_{4}, f\right)+\bar{N}\left(r, a_{4}, g\right)=2 \bar{N}_{0}\left(r, a_{4}, f, g\right)+\bar{N}_{12}\left(r, a_{4}, f, g\right),
$$

from this and (2.5) we obtain (2.6), and Lemma 2.2 is thus proved.

\section{Preparatory theorems}

Using Lemma 2.2, we can prove the following theorems:

THEOREM 4.1. Let $f$ and $g$ be nonconstant entire functions and let $a_{j}(j=1$, $2,3,4)$ be four distinct elements in $S(f) \cap S(g)$. If $f \neq \equiv g$, then

$$
2 T(r, f)+2 T(r, g) \leq 7 \sum_{j=1}^{4} \bar{N}_{12}\left(r, a_{j}, f, g\right)+S(r, f)+S(r, g) .
$$

Proof. By Lemma 2.2 we have

$$
\begin{aligned}
\bar{N}\left(r, a_{4}, f\right)+\bar{N}\left(r, a_{4}, g\right) \leq & 2 \sum_{j=1}^{4} \bar{N}_{12}\left(r, a_{j}, f, g\right)-\bar{N}_{12}\left(r, a_{4}, f, g\right) \\
& +S(r, f)+S(r, g) .
\end{aligned}
$$

In the same manner as above, we have

$$
\begin{aligned}
\bar{N}\left(r, a_{3}, f\right)+\bar{N}\left(r, a_{3}, g\right) \leq & 2 \sum_{j=1}^{4} \bar{N}_{12}\left(r, a_{j}, f, g\right)-\bar{N}_{12}\left(r, a_{3}, f, g\right) \\
& +S(r, f)+S(r, g), \\
\bar{N}\left(r, a_{2}, f\right)+\bar{N}\left(r, a_{2}, g\right) \leq & 2 \sum_{j=1}^{4} \bar{N}_{12}\left(r, a_{j}, f, g\right)-\bar{N}_{12}\left(r, a_{2}, f, g\right) \\
& +S(r, f)+S(r, g), \\
\bar{N}\left(r, a_{1}, f\right)+\bar{N}\left(r, a_{1}, g\right) \leq & 2 \sum_{j=1}^{4} \bar{N}_{12}\left(r, a_{j}, f, g\right)-\bar{N}_{12}\left(r, a_{1}, f, g\right) \\
& +S(r, f)+S(r, g) .
\end{aligned}
$$


By Lemma 2.6, from (4.2), (4.3), (4.4) and (4.5) we obtain (4.1), and Theorem 4.1 is thus proved.

THEOREM 4.2. Let $f$ and $g$ be nonconstant entire functions and let $a_{j}(j=1$, $2,3,4)$ be four distinct elements in $S(f) \cap S(g)$. If $f$ and $g$ share $a_{1}$ "IM", and $f \not \equiv g$, then

$$
\frac{3}{2} T(r, f)+\frac{3}{2} T(r, g) \leq 5 \sum_{j=2}^{4} \bar{N}_{12}\left(r, a_{j}, f, g\right)+S(r, f)+S(r, g) .
$$

Proof. By Lemma 2.2 we may obtain (4.2), (4.3) and (4.4). Noting that $f$ and $g$ share $a_{1}$ "IM", from (4.2), (4.3) and (4.4) we have for $i=2,3,4$

$$
\begin{aligned}
\bar{N}\left(r, a_{i}, f\right)+\bar{N}\left(r, a_{i}, g\right) \leq & 2 \sum_{j=2}^{4} \bar{N}_{12}\left(r, a_{j}, f, g\right)-\bar{N}_{12}\left(r, a_{i}, f, g\right) \\
& +S(r, f)+S(r, g) .
\end{aligned}
$$

By Lemma 2.5, from (4.7) we obtain (4.6).

THEOREM 4.3. Let $f$ and $g$ be nonconstant entire functions and let $a_{j}(j=1$, $2,3,4)$ be four distinct elements in $S(f) \cap S(g)$. If $f$ and $g$ share $a_{1} a_{2}$ "IM", and $f \not \equiv g$, then

$$
T(r, f)+T(r, g) \leq 3 \sum_{j=3}^{4} \bar{N}_{12}\left(r, a_{j}, f, g\right)+S(r, f)+S(r, g) .
$$

Proof. By Lemma 2.2 we obtain (4.2) and (4.3). Noting that $f$ and $g$ share $a_{1} \quad a_{2}$ "IM", from (4.2) and (4.3) we have for $i=3,4$

$$
\begin{gathered}
\bar{N}\left(r, a_{i}, f\right)+\bar{N}\left(r, a_{i}, g\right) \leq \\
2 \sum_{j=3}^{4} \bar{N}_{12}\left(r, a_{j}, f, g\right)-\bar{N}_{12}\left(r, a_{i}, f, g\right) \\
+S(r, f)+S(r, g) .
\end{gathered}
$$

By Lemma 2.4, from (4.9) we obtain (4.8).

THEOREM 4.4. Let $f$ and $g$ be nonconstant entire functions and let $a_{j}(j=1$, $2,3,4)$ be four distinct elements in $S(f) \cap S(g)$. If $f$ and $g$ share $a_{1}, a_{2}, a_{3}$ "IM", and $f \not \equiv g$, then

$$
T(r, f)+T(r, g) \leq 3 \bar{N}_{12}\left(r, a_{4}, f, g\right)+S(r, f)+S(r, g) .
$$

Proof. Noting that $f$ and $g$ share $a_{3}$ "IM", from Theorem 4.3 we obtain (4.10). 


\section{Proof of main results}

\subsection{Proof of Theorem 1.1}

Suppose that $f \not \equiv g$. By Theorem 4.1, we can obtain (4.1).

If $k=\infty$, from (1.3) we have

$$
\bar{N}_{12}\left(r, a_{j}, f, g\right)=0 \quad(j=1,2,3,4) .
$$

Substituting (5.1) into (4.1) we obtain

$$
2 T(r, f)+2 T(r, g)=S(r, f)+S(r, g),
$$

which is a contradiction. Next, we assume that $k$ is a positive integer.

Noting (1.3), we have

$$
\bar{N}_{12}\left(r, a_{j}, f, g\right) \leq \bar{N}_{(k+1}\left(r, a_{j}, f\right)+\bar{N}_{(k+1}\left(r, a_{j}, g\right) \quad(j=1,2,3,4) .
$$

Substituting (5.2) into (4.1) we obtain

$$
\begin{aligned}
2 T(r, f)+2 T(r, g) \leq & 7 \sum_{j=1}^{4} \bar{N}_{(k+1}\left(r, a_{j}, f\right)+7 \sum_{j=1}^{4} \bar{N}_{(k+1}\left(r, a_{j}, g\right) \\
& +S(r, f)+S(r, g) .
\end{aligned}
$$

By Lemma 2.6, we have

$$
\begin{aligned}
& \sum_{j=1}^{4} \bar{N}_{(k+1}\left(r, a_{j}, f\right) \leq \frac{2}{k} T(r, f)+S(r, f), \\
& \sum_{j=1}^{4} \bar{N}_{(k+1}\left(r, a_{j}, g\right) \leq \frac{2}{k} T(r, g)+S(r, g) .
\end{aligned}
$$

Substituting (5.4) and (5.5) into (5.3) we obtain

$$
2 T(r, f)+2 T(r, g) \leq \frac{14}{k} T(r, f)+\frac{14}{k} T(r, g)+S(r, f)+S(r, g),
$$

which contradicts the assumption $k \geq 8$. Thus, $f \equiv g$.

\subsection{Proof of Theorem 1.2}

Suppose that $f \not \equiv g$. By Theorem 4.4, we can obtain (4.10). Noting (1.4), we have

$$
\begin{aligned}
\bar{N}_{12}\left(r, a_{4}, f, g\right) & \leq \bar{N}_{(k+1}\left(r, a_{4}, f\right)+\bar{N}_{(k+1}\left(r, a_{4}, g\right) \\
& \leq \frac{1}{k+1} T(r, f)+\frac{1}{k+1} T(r, g)+S(r, f)+S(r, g) .
\end{aligned}
$$

Substituting (5.6) into (4.10) we obtain

$$
T(r, f)+T(r, g) \leq \frac{3}{k+1} T(r, f)+\frac{3}{k+1} T(r, g)+S(r, f)+S(r, g),
$$

which contradicts the assumption $k \geq 3$. Thus, $f \equiv g$. 


\subsection{Proof of Theorem 1.3}

we get

Suppose that $f \not \equiv g$. By Theorem 4.4, we can obtain (4.10). From (4.10),

$$
\lambda\left(a_{4}, f, g\right) \leq \frac{2}{3},
$$

which contradicts (1.5). Thus, $f \equiv g$.

\section{Supplement to main results}

Proceeding as in the proof of Theorem 1.1, by Theorem 4.2, 4.3 respectively, we have the following theorems, which are improvements of Theorem A.

THEOREM 6.1. Let $f$ and $g$ be nonconstant entire functions and let $a_{j}(j=1$, $2,3,4)$ be four distinct elements in $S(f) \cap S(g)$. If $f$ and $g$ share $a_{1}$ "IM", and

$$
\bar{E}\left(a_{j}, k, f\right)=\bar{E}\left(a_{j}, k, g\right) \quad(j=2,3,4),
$$

where $k$ is a positive integer or $\infty$ with $k \geq 6$, then $f \equiv g$.

THEOREM 6.2. Let $f$ and $g$ be nonconstant entire functions and let $a_{j}(j=1$, $2,3,4)$ be four distinct elements in $S(f) \cap S(g)$. If $f$ and $g$ share $a_{1}$ and $a_{2}$ "IM", and

$$
\bar{E}\left(a_{j}, k, f\right)=\bar{E}\left(a_{j}, k, g\right) \quad(j=3,4),
$$

where $k$ is a positive integer or $\infty$ with $k \geq 4$, then $f \equiv g$.

Proceeding as in the proof of Theorem 1.3, by Theorem 4.1, 4.2, 4.3 respectively, we have the following theorems, which are improvements of Theorem A.

THEOREM 6.3. Let $f$ and $g$ be nonconstant entire functions and let $a_{j}(j=1$, $2,3,4)$ be four distinct elements in $S(f) \cap S(g)$. If

$$
\sum_{j=1}^{4} \lambda\left(a_{j}, f, g\right)>\frac{26}{7},
$$

then $f \equiv g$.

THEOREM 6.4. Let $f$ and $g$ be nonconstant entire functions and let $a_{j}(j=1$, $2,3,4)$ be four distinct elements in $S(f) \cap S(g)$. If $f$ and $g$ share $a_{1}$ "IM", and

$$
\sum_{j=2}^{4} \lambda\left(a_{j}, f, g\right)>\frac{27}{10},
$$

then $f \equiv g$. 
THEOREM 6.5. Let $f$ and $g$ be nonconstant entire functions and let $a_{j}(j=1$, $2,3,4)$ be four distinct elements in $S(f) \cap S(g)$. If $f$ and $g$ share $a_{1}$ and $a_{2}$ "IM", and

$$
\lambda\left(a_{3}, f, g\right)+\lambda\left(a_{4}, f, g\right)>\frac{5}{3},
$$

then $f \equiv g$.

Acknowledgement. The author wants to thank the referee for valuable comments.

\section{REFERENCES}

[1] W. K. Hayman, Meromorphic Functions, Oxford Math. Monogr., Clarendon Press, Oxford, 1964.

[2] K. IshizAKI AND N. Toda, Unicity theorems for meromorphic functions sharing four small functions, Kodai Math. J., 21 (1998), 350-371.

[ 3 ] H. X. YI, On results of Gopalakrishna-Bhoosnurmath and Singh-Dhar, Indian J. Pure Appl. Math., 30 (1999), 1081-1089.

[4] R. Nevanlinna, Le Théorème de Picard-Borel et la Thèorie des Fonctions Méromorphes, Gauthier-Villars, Paris, 1929.

[ 5 ] H. X. Yi AND C. C. YANG, Uniqueness Theory of Meromorphic Functions, Pure and Applied Math. Monogr. 32, Science Press, Beijing, 1995.

[6] B. Q. LI, Uniqueness of entire functions sharing four small functions, Amer. J. Math., 119 (1997), 841-858.

[ 7 ] Y. H. LI, Entire functions that share four finite slowly growth meromorphic functions IM, Acta Math. Sinica, 41 (1998), 249-260.

Department of Mathematics

SHANDONG UNIVERSITY

JINAN 250100

P. R. CHINA

e-mail: hxyi@sdu.edu.cn 